\title{
Superior Semicircular Canal Dehiscence Mimicking Otosclerotic Hearing Loss
}

\author{
Saumil N. Merchant ${ }^{\mathrm{a}-\mathrm{c}}$, John J. Rosowski ${ }^{\mathrm{a}-\mathrm{c}}$, Michael J. McKenna ${ }^{\mathrm{a}-\mathrm{c}}$ \\ aDepartment of Otology and Laryngology, Harvard Medical School, bepartment of \\ Otolaryngology, Massachusetts Eye and Ear Infirmary, Boston, ${ }^{\mathrm{c} D i v i s i o n}$ of Speech \\ and Hearing Science Technology, Massachusetts Institute of Technology, Cambridge, \\ Mass., USA
}

\begin{abstract}
A puzzling aspect of middle ear surgery is the presence of an air-bone gap in a small number of cases with no apparent cause. We believe that some of these cases are due to unrecognized superior semicircular canal dehiscence (SSCD). We have now gathered experience from 20 patients with SSCD presenting with apparent conductive hearing loss without vestibular symptoms. All affected ears had SSCD on high-resolution CT scan. The common findings in these patients were: (1) the air-bone gaps occurred in the lower frequencies below $2,000 \mathrm{~Hz}$, and ranged from 10 to $60 \mathrm{~dB} ;(2)$ bone conduction thresholds below $2,000 \mathrm{~Hz}$ were sometimes negative $(-5 \mathrm{~dB}$ to $-15 \mathrm{~dB})$; (3) the acoustic (stapedial) reflex was present; (4) measurement of umbo velocity by laser Doppler vibrometry showed slight hypermobility of umbo motion; (5) the vestibular-evoked myogenic potential response was present, with thresholds that were abnormally low, and (6) the middle ear was normal at exploratory tympanotomy, including normal mobility of the ossicles and a patent round window niche. We have investigated the mechanism of the air-bone gap due to SSCD using a theoretical framework, clinical research data and an animal model (chinchilla). Our research supports the hypothesis that SSCD introduces a 'third' window into the inner ear which produces the airbone gap by (1) shunting air-conducted sound away from the cochlea, thus elevating air conduction thresholds, and (2) increasing the difference in impedance between the scala tympani and scala vestibuli, thus improving thresholds for bone-conducted sound.
\end{abstract}

Copyright (C) 2007 S. Karger AG, Basel

Superior semicircular canal dehiscence (SSCD) syndrome is a recently described clinical entity associated with vestibular symptoms typically evoked by sound and pressure stimuli $[1,2]$. As experience with this syndrome has accrued, it has become apparent that some patients with SSCD can present with 
apparent conductive hearing loss without vestibular symptoms [3-5]. In many cases, the clinical presentation so closely mimics that of otosclerosis that exploratory tympanotomy and even stapedectomy has been performed on some of these patients without benefit to the hearing [3-5]. In this paper, we describe our experience with this condition, report on our research to understand the mechanisms of the hearing loss, and point out methods that may enable the clinician to make an accurate diagnosis.

\section{Clinical Presentation of SSCD with Conductive Hearing Loss}

In the 3 years between 2001 and 2004, we have made the diagnosis of SSCD as a cause of apparent conductive hearing loss without vertigo in 20 cases. These patients have ranged from 20 to 70 years in age, equally divided between males and females. The typical clinical presentation is that of an adult patient complaining of a unilateral or bilateral progressive hearing loss. Some patients also complain of autophony, aural blockage or distortion of sounds. When asked specifically about vestibular complaints, about $20 \%$ volunteered a history of mild disequilibrium or imbalance. Otoscopy typically reveals a normal tympanic membrane with an aerated middle ear. The Rinne test is negative and the Weber test lateralizes to the affected ear. In our series, only $20 \%$ of patients had sound-induced or pressure-induced nystagmus when tested with video Frenzel lenses.

Audiologic testing reveals an air-bone gap which is greater in the lower frequencies (fig. 1). The air-bone gaps can be quite large, up to $40-50 \mathrm{~dB}$. Bone conduction thresholds are often negative $(-5 \mathrm{~dB}$ to $-15 \mathrm{~dB})$ below $2,000 \mathrm{~Hz}$ in many cases. However, in some patients in our series, the bone thresholds were not negative and even a Carhart notch was observed. The acoustic (stapedial) reflex is generally present in SSCD cases, whereas it is absent in middle ear diseases such as otosclerosis. The vestibular-evoked myogenic potential (VEMP) response is generally present in SSCD with thresholds that are abnormally low $[5,6]$ (in contrast, the VEMP response is usually absent in otosclerotic hearing loss). Measurement of umbo velocity by laser Doppler vibrometry shows the velocity to be in the high normal range in SSCD, approximately one standard deviation above mean normal $[7,8]$. In otosclerotic hearing loss, the umbo velocity is usually one standard deviation below mean normal [9]. Exploratory tympanotomy is negative, with normal mobility of all ossicles and a patent round window. The diagnosis is made by high-resolution computed tomographic (CT) scanning with reformatting of the images in planes parallel to and perpendicular to that of the superior canal $[1,2,5,10]$. 


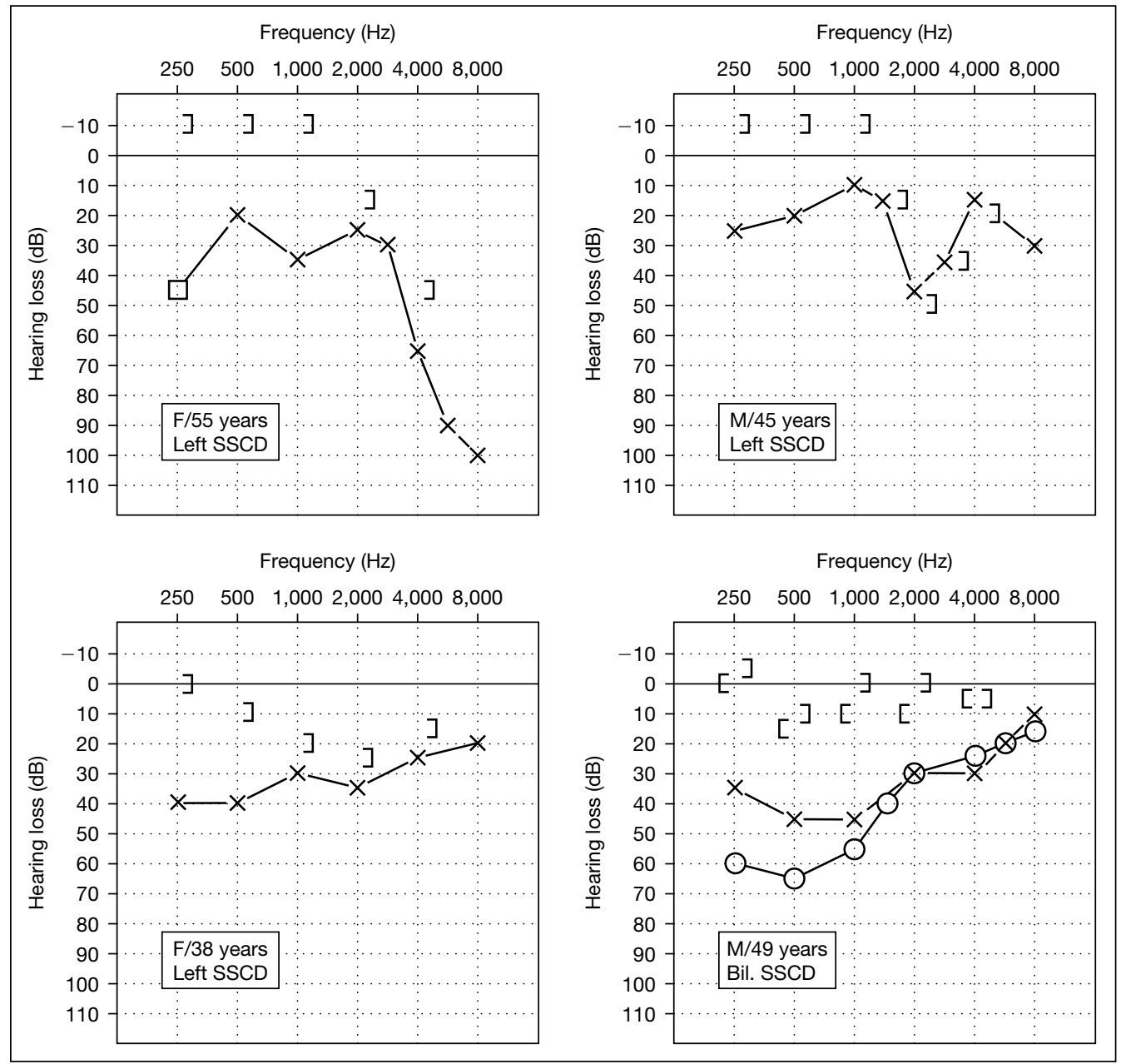

Fig. 1. Audiogram of 4 of our cases with SSCD presenting as conductive hearing loss without vertigo. The air-bone gaps are larger in the lower frequencies. Note that the bone conduction thresholds are sometimes better than $0 \mathrm{~dB}(-5$ to $-15 \mathrm{~dB})$ in the lower frequencies.

\section{Evidence that SSCD Can Cause a Conductive Hearing Loss}

Direct evidence that SSCD can result in an air-bone gap is the improvement of hearing and resolution of the gap after surgical closure of an SSCD. We have experience with one patient, a 50-year-old man who had a low-frequency air-bone gap of $25 \mathrm{~dB}$ and chronic disequilibrium due to SSCD (fig. 2). He underwent a 


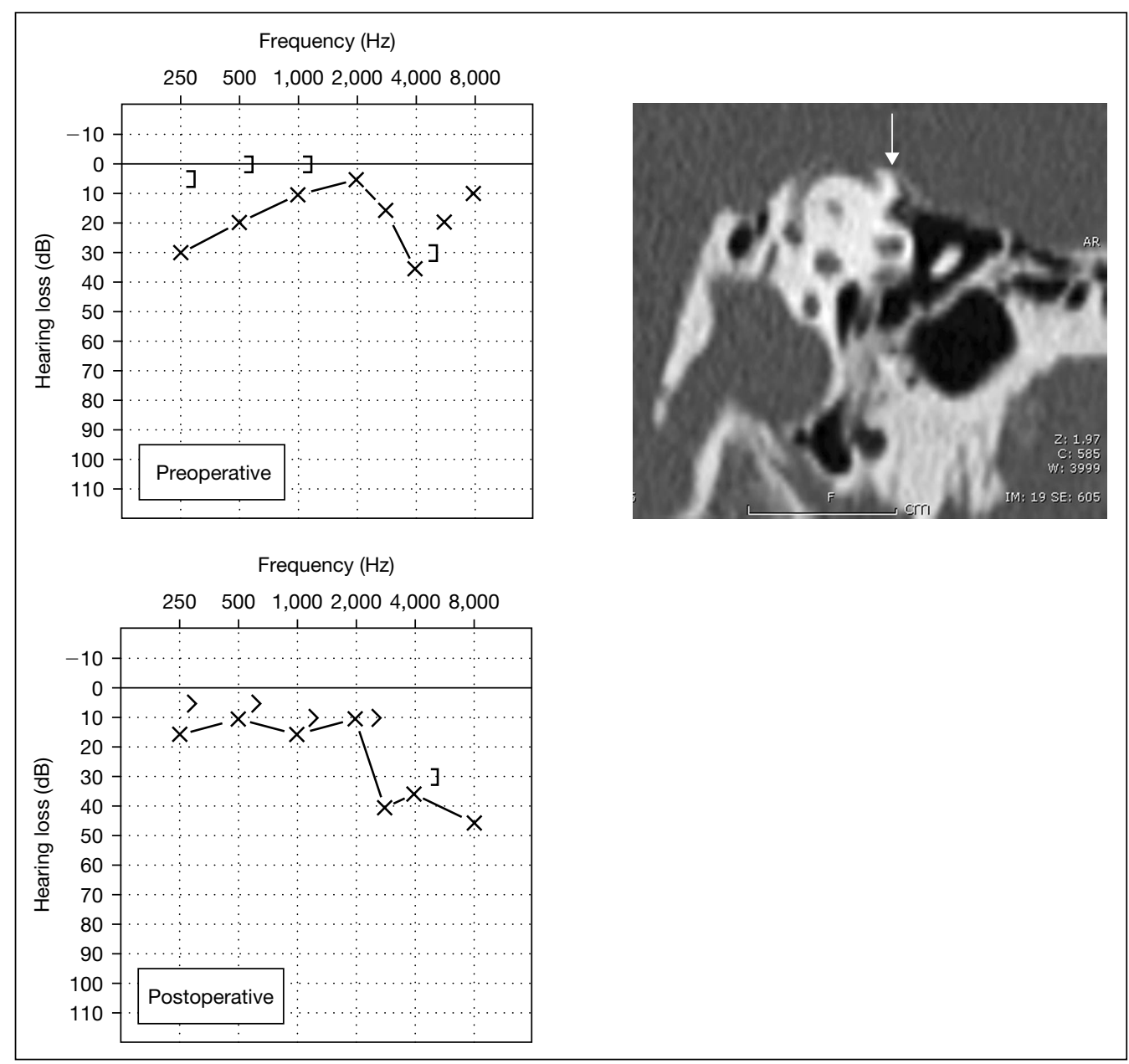

Fig. 2. A case of a 50-year-old man with conductive hearing loss and chronic disequilibrium due to SSCD. The preoperative audiogram shows a low-frequency air-bone gap of up to $25 \mathrm{~dB}$. The CT scan image shows the dehiscence (arrow). Middle fossa plugging was done with bone wax and temporalis fascia. The audiogram 2 weeks after surgery shows resolution of the air-bone gap, with a high-frequency sensorineural loss at $8 \mathrm{kHz}$.

middle fossa procedure with plugging of the SSCD using bone wax and temporalis fascia. His vestibular complaints resolved and a postoperative audiogram showed closure of the air-bone gap. Minor et al. [3] described a patient with a significant conductive hearing loss and oscillopsia who underwent resurfacing of a dehiscent superior canal, and who experienced improvement of the air conduction 


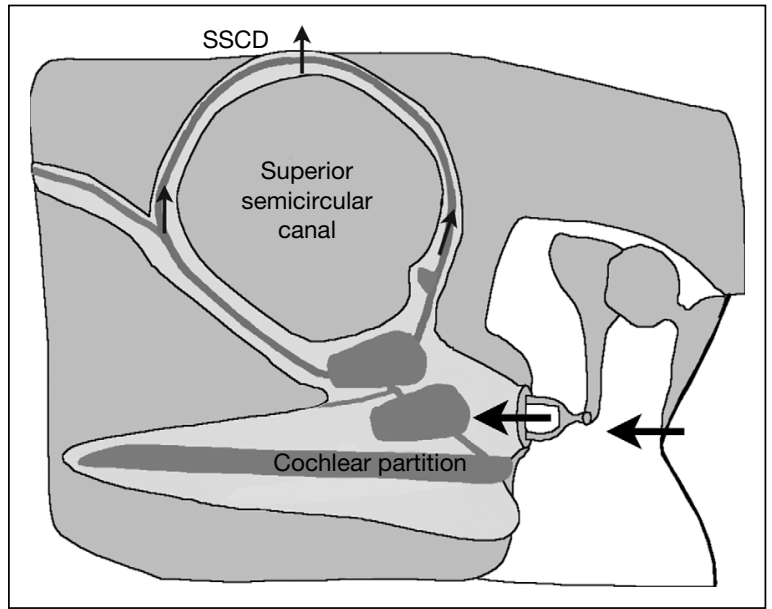

Fig. 3. Proposed mechanism of hearing loss by air conduction due to SSCD. Acoustic energy is shunted through the dehiscence away from the cochlea.

thresholds by $20 \mathrm{~dB}$. In addition, it has been noted that patients with SSCD and the typical vestibular complaints often have a small, low-frequency air-bone gap of 5-10 dB [1, 2, 5]. This small conductive loss usually resolves after surgical repair of the SSCD [5].

There are indirect lines of evidence to support the hypothesis that SSCD can cause a conductive hearing loss: (1) middle ear exploratory surgery in these cases does not reveal any abnormality to explain the conductive hearing loss, and (2) diagnostic tests such as the acoustic reflex, VEMP response and laser Doppler vibrometry of the umbo show results that are the opposite of what is expected in the presence of middle ear pathology.

\section{Mechanisms of Conductive Hearing Loss Resulting from SSCD}

We have initiated a series of studies to investigate the mechanism by which an air-bone gap could arise as a result of an SSCD, including mathematical model analyses, experiments in a chinchilla model of SSCD, and measurements of middle ear function using clinical research tools such as laser Doppler vibrometry in patients with SSCD [7]. The evidence to date from our investigations supports the hypothesis that SSCD can cause a conductive hearing loss by acting as a 'third' window in the inner ear $[3,5,7]$ that results in elevation of thresholds for air-conducted sounds and reduction of thresholds for boneconducted sounds. As shown in figure 3, air-conducted sound stimuli enter the 
vestibule through motion of the stapes. Our data support the hypothesis that the SSCD shunts a portion of this acoustic energy through the dehiscence away from the cochlea, thus resulting in a hearing loss by air conduction. In our model calculations, the physical constraints imposed by the anatomical dimensions of the human labyrinth indicate that such hearing loss will be greatest at the lower frequencies. The effect of an SSCD on bone conduction can be understood based on the compressional mechanism of bone conduction. As shown in figure $4 \mathrm{a}$, in the normal ear, compression of inner ear fluid by a bone-conducted sound stimulates the cochlea because of an inequality in the impedance on the two sides of the cochlear partition, where the scala tympani impedance is dominated by the round window and the scala vestibuli impedance is dominated by the stapes and oval window. This inequality leads to a pressure difference across the cochlear partition, resulting in motion of the basilar membrane which leads to perception of bone-conducted sound. As shown in figure $4 \mathrm{~b}$, we hypothesize that the SSCD, acting as a shunt at low frequencies, increases the inequality in the impedance between the two scalae, thereby increasing the cochlea's response to compressional bone conduction. Such a mechanism can explain the 'negative' bone conduction thresholds observed in our patients. Again, the physical dimensions of the human labyrinth would constrain the improvement in bone conduction thresholds to frequencies below $2,000 \mathrm{~Hz}$, which is consistent with our clinical observations. We believe that a 'third' window hypothesis as described above can also explain the occurrence of a low-frequency air-bone gap in other types of inner ear pathologies such as an enlarged vestibular aqueduct [11] or dehiscence of other semicircular canals.

\section{Differentiation of Conductive Hearing Loss due to SSCD from Otosclerosis}

The typical features of conductive hearing loss in SSCD are a low-frequency air-bone gap with bone conduction thresholds that are better than $0 \mathrm{~dB}$ in the lower frequencies. It is important to accurately determine the audiometric bone conduction thresholds by testing at levels below $0 \mathrm{~dB}$ HL, if necessary. Acoustic reflexes are generally present in SSCD but absent in otosclerotic stapes fixation. If available, VEMP testing and laser Doppler vibrometry testing can also assist in the diagnosis, as previously described. In suspected cases of SSCD, the diagnosis can be made by high-resolution CT scanning with reformatting of the images in planes parallel to and perpendicular to that of the superior canal. The recognition and characterization of conductive hearing loss in SSCD combined with a heightened awareness of its diagnostic possibility should serve to reduce the number of patients who undergo unnecessary middle ear surgery in the future. 


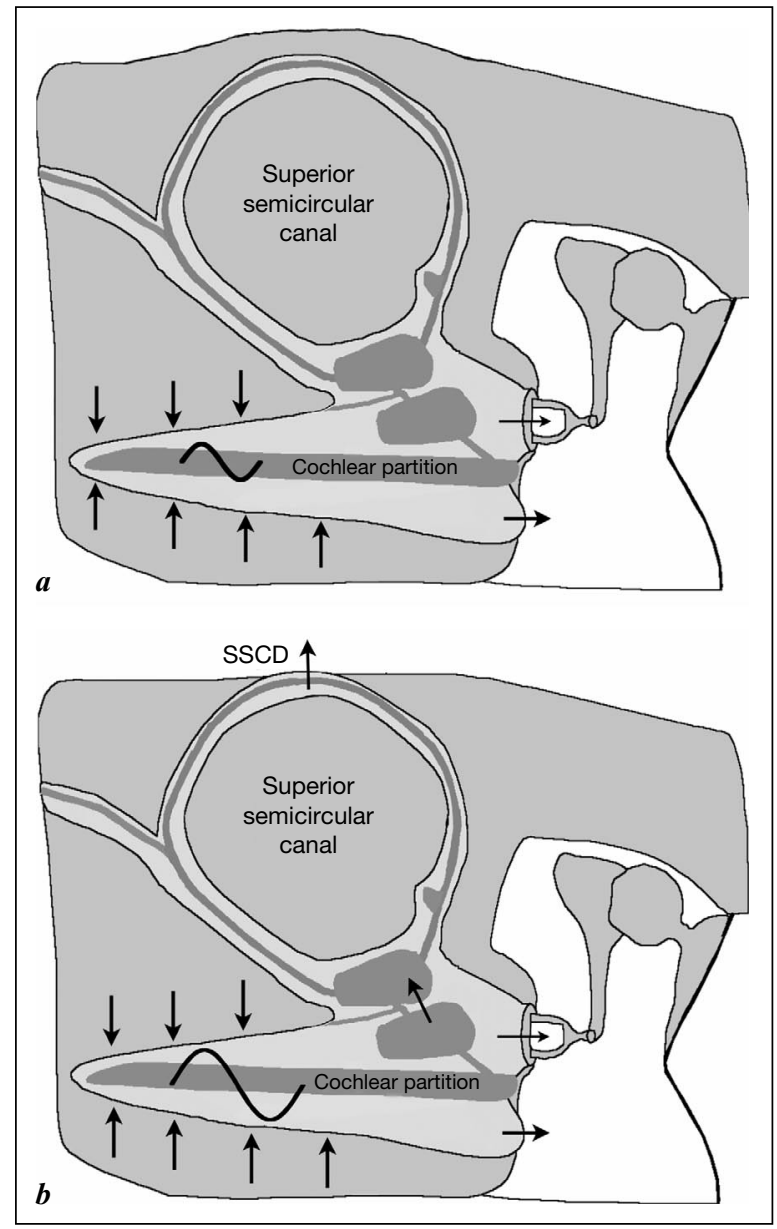

Fig. 4. $a$ Normal bone conduction by the compressional mechanism. Bone-conducted sound causes compression of inner ear fluids. This results in a pressure difference across the cochlear partition because of unequal impedance between the scala tympani and scala vestibuli. In the normal ear, the scala tympani impedance is controlled by the round window, while the scala vestibuli impedance is controlled by the stapes and oval window. $\boldsymbol{b}$ Proposed mechanism of improved bone conduction thresholds in SSCD. SSCD acts as a third window and increases the inequality in impedance between the scalae. Hence, the pressure difference across the cochlear partition is increased compared to normal, resulting in improved thresholds for bone-conducted sound. 


\section{Future Directions}

So far, we have not performed surgical repair of SSCD in patients with conductive hearing losses without disabling vertigo because of the risk of sensorineural deafness as well as the potential risks of middle fossa surgery. We have repaired SSCD for relief of vestibular complaints via a middle fossa approach in 12 patients. Although no case developed a severe or profound hearing loss in our series, a mild to moderate high-frequency sensorineural hearing loss at 4 and $8 \mathrm{kHz}$ occurred in 2 of our 12 cases. Our philosophy and recommendations may change as we accumulate more experience with surgical management of this condition.

The natural history and etiology of SSCD remain uncertain. It is interesting to note that 2 cases in our series with conductive hearing loss without vertigo are brothers, each with bilateral SSCD, raising the possibility of a genetic predisposition to SSCD. We also do not understand why some patients with SSCD become symptomatic with primarily vestibular complaints, whereas others present with both auditory and vestibular symptoms, and a third group has only auditory symptoms. We are hopeful that ongoing clinical and basic research will clarify some of these issues in the future.

\section{Acknowledgement}

This work was supported by NIH grant R01 DC04798.

\section{References}

1 Minor LB, Solomon D, Zinreich JS, Zee DS: Sound- and/or pressure-induced vertigo due to bone dehiscence of the superior semicircular canal. Arch Otolaryngol Head Neck Surg 1998;124: 249-258.

2 Minor LB: Superior canal dehiscence syndrome. Am J Otol 2000;21:9-19.

3 Minor LB, Carey JP, Cremer PD, Lustig LR, Streubel SO, Ruckenstein MJ: Dehiscence of bone overlying the superior canal as a cause of apparent conductive hearing loss. Otol Neurotol $2003 ; 24: 270-278$.

4 Halmagyi GM, Aw ST, McGarvie LA, Todd MJ, Bradshaw A, Yavor RA, Fagan PA: Superior semicircular canal dehiscence simulating otosclerosis. J Laryngol Otol 2003;117:553-557.

5 Mikulec AA, McKenna MJ, Ramsey MJ, Rosowski JJ, Herrmann BS, Rauch SD, Curtin HD, Merchant SN: Superior semicircular canal dehiscence presenting as conductive hearing loss without vertigo. Otol Neurotol 2004;25:121-129.

6 Streubel SO, Cremer PD, Carey JP, Weg N, Minor LB: Vestibular-evoked myogenic potentials in the diagnosis of superior canal dehiscence syndrome. Acta Otolaryngol Suppl 2001;545:41-49.

7 Rosowski JJ, Songer JE, Nakajima HH, Brinsko KM, Merchant SN: Clinical, experimental and theoretical investigations of the effect of superior semicircular canal dehiscence on hearing mechanisms. Otol Neurotol 2004;25:323-332. 
8 Whittemore KR, Merchant SN, Poon BB, Rosowski JJ: A normative study of tympanic membrane motion in humans using a laser Doppler vibrometer. Hear Res 2004;187:85-104.

9 Rosowski JJ, Mehta RP, Merchant SN: Diagnostic utility of laser-Doppler vibrometry in conductive hearing loss with normal tympanic membrane. Otol Neurotol 2003;24:165-175.

10 Belden CJ, Weg N, Minor LB, Zinreich SJ: CT evaluation of bone dehiscence of the superior semicircular canal as a cause of sound- and/or pressure-induced vertigo. Radiology 2003;226: 337-343.

11 Nakashima T, Ueda H, Furuhashi A, Sato E, Asahi K, Naganawa S, Beppu R: Air-bone gap and resonant frequency in large vestibular aqueduct syndrome. Am J Otol 2000;21:671-674.

Saumil N. Merchant, MD

Department of Otolaryngology

Massachusetts Eye and Ear Infirmary, 243 Charles Street

Boston, MA 02114-3096 (USA)

Tel. +1 617573 3503, Fax +1 617573 3939, E-Mail saumit_merchant@meei.harvard.edu 